\section{GP2 \\ HEAD INJURIES IN CHILDREN UNDER 1 YEAR IN CHILDREN'S UNIVERSITY HOSPITAL, TEMPLE STREET}

Darragh 0 Shea*, Conor Hensey, Patrick Fitzpatrick. Children's University Hospital, Temple Street, Dublin, Ireland

10.1136/archdischild-2019-epa.69

Introduction Head injury in children is a common presentation to emergency departments (ED). Specific age groups are difficult to assess, particularly children $<1$ year of age. This leads to a high rate of admission even when not clinically indicated. The aim of this study was to evaluate our current practice and establish an evidence base to direct future guidelines on head injury admissions.

Methods A retrospective audit of all children $<1$ year admitted to Temple Street for head injury in 2017 was undertaken. Data was recorded from the ED 'Symphony' system and HIPE coding, including age, mechanism of injury, neuro-imaging (NICE criteria, modality, timing, findings), neurosurgical intervention, child protection concerns (CPC), outcomes and follow-up.

Results Study population was 403, 96 of which were admitted. The median age was 3 months. The most common mechanism of injury was fall from a bed/couch/mat (26\%) followed by fall from a carrier seat unrestrained $(21.8 \%)$.

13 had a CT head (CTH) all meeting NICE criteria. 8 had a skull $\mathrm{x}$-ray (SXR), 5 of which required CTH. 2 additional infants met criteria for CTH but didn't have one (as GCS rapidly improved). All $\mathrm{CTH}$ were abnormal with skull fractures identified. 3 required neurosurgical intervention. All 13 were followed in neurosurgical clinic. Neuro-observations ranged from 4-24 hours in patients not requiring CTH.

Initial CPC were raised in 9 cases with medical social work (MSW) involvement in all cases. 6 Skeletal surveys (SS) were performed; 4 Non-accidental injury (NAI) work-up and 2 following a dangerous mechanism of injury. 11 patients represented with a further head injury. In 2, CPC were noted with MSW involvement, with one further SS.

Discussion With no indications for CTH or CPC, neuro-observations are performed up to 24hours. With early senior involvement this can be as short as 4hours. Although NICE gives no specific duration for neuro-observations, with our consistently good outcomes and low representation rates, it may be safe to shorten admissions.

ED staff recognised CPC in all cases, made a MSW referral and flagged this to the admitting teams. 5 referrals progressed to a full NAI workup with no additional injuries or concerns noted on MSW assessment. No admitted cases proceeded to CP case conference. This indicates that our paediatric ED staff are aware of and are looking for potential CP red flags. Further prospective study of patients discharged following observation in ED is indicated.

\section{GP3 'FORGOTTEN BABY SYNDROME': A SYSTEMATIC REVIEW AND ANALYSIS OF CAREGIVER INTENTION}

${ }^{1}$ Richard Lee-Kelland*, ${ }^{2}$ Fiona Finlay. ${ }^{1}$ Sirona Healthcare, Bristol, UK; ${ }^{2}$ Virgincare, Bath, UK

10.1136/archdischild-2019-epa.70

Aims Each year around 37 children in the US alone are killed by heatstroke through being left unattended in vehicles. In the past, caregivers of these children have been charged with neglect or manslaughter. In recent cases such as Noah Zunde (2017), inquests have drawn on expert witnesses who have expanded on a psychological basis for these cases, pointing at an 'overriding' habitual memory i.e. the routine of taking a child to playgroup. The term 'forgotten baby syndrome' has come to prominence to describe a scenario whereby a child comes to injury by being unintentionally left in a car. The implication being that a parent could be acquitted of manslaughter if the child was truly forgotten. This may have far reaching consequences for defining neglect. We completed a literature review to assess the proportion of unintentional vs intentional cases where a child was injured by heatstroke by being left unattended in a vehicle.

Methods A systematic review of the literature was completed on Medline. In addition data was taken from the US national safety council via noheatstroke.org.

Results The USA national safety council reviewed 743 child deaths from heatstroke after being left unattended in a vehicle from 1998-2017 in 54\% the child was unintentionally left, $27 \%$ the child gained access to the vehicle, $18 \%$ the child was intentionally left and $1 \%$ circumstances were unknown. A Brazilian study (Costa 2016) identified 31 cases (20 fatal) in which $71 \%$ were unintentional, 3\% child gained access, 23\% were intentional and 3\% unknown. A small Italian study (Ferrara 2013) identified 16 cases (2 fatalities) of which $18 \%$ were unintentional, $75 \%$ were intentional and $6 \%$ unknown.

Conclusion Most children who die through being left unattended are reported to have been left in vehicles unintentionally. Further research is required to establish what initiatives work, if most cases are truly unintentional, public health strategies that merely explain the dangers of leaving a child unattended such as the American, 'It Can Happen' are less likely to be successful - parents may be aware of the danger, but simply forget the child is in the vehicle. Future interventions that are universal such 'child reminder systems' in cars may be more successful. The difference between 'forgotten baby syndrome' and neglect remains difficult to define.

\section{GP4 DE-LABELLING BETA-LACTAM ALLERGY IN CHILDREN IN AN OUTPATIENT SETTING USING A SINGLE DOSE PROTOCOL}

${ }^{1,2}$ Hilary Allen*, ${ }^{3}$ Marta Vazquez-Ortiz, ${ }^{4}$ Andrew Murphy, ${ }^{1,5}$ Edina Moylett. ${ }^{1}$ Department of Paediatrics. University Hospital, Galway, Ireland; '2Department of Medicine. Imperial College, London, UK; ${ }^{3}$ Department of Paediatrics. Imperial College, London, UK; ${ }^{4}$ Department of General Practice and HRB Clinical Trial Network Primary Care Ireland. National University of Ireland, Galway, Ireland; ${ }^{5}$ Department of Paediatrics. National University of Ireland, Galway, Ireland

\subsection{6/archdischild-2019-epa.71}

Background and aims Up to 5\% of children report a reaction to a beta-lactam antibiotic. This is commonly a rash which occurs in the setting of a viral illness. These children are often labelled with a drug allergy.

Previous studies on drug allergy in children have involved skin prick testing, intradermal testing and graded dose challenges in the hospital setting. This study was conducted to explore the diagnosis of drug allergy in a selected low-risk cohort of 'beta-lactam allergy labelled' children, using risk stratified, single dose, oral beta-lactam challenges, potentially reproducible in primary care. 